\title{
IMPROVISATION OF AN OPTICAL PRESSURE SENSOR BASED DYNAMIC FOOT PRESSURE MEASUREMENT SYSTEM
}

\author{
K Siddique-e Rabbani, ${ }^{1,2}$, SM Zahid Ishraque ${ }^{2}$, M Shahedul Islam², Rhaad Muasir \\ Rabbani $^{2}$ \\ ${ }^{1}$ Department of Biomedical Physics \& Technology, University of Dhaka, Dhaka-1000 \\ ${ }^{2}$ Bangladesh Institute for Biomedical Engineering \& Appropriate Technology (BIBEAT) \\ rabbani@univdhaka.edu, zahidishraque@gmail.com,riyad_csedu@yahoo.com,rhaadmrabbani@gmail.com
}

\begin{abstract}
Dynamic foot pressure measurement is necessary particularly for diabetic patients to prevent ulcers, eventually leading to gangrene and amputation. The present work reports a low cost optical sensing system for the above, suitable for the Third World. It uses a horizontally placed transparent Acrylic plate with a webcam placed below facing upwards. A white paper, backed by a black adhesive plastic sheet, covered the whole plate on the top. Light from a tubular fluorescent lamp entered the acrylic plate from a side and traversed the thickness through total internal reflection. At points of pressure applied from the top, the air between the paper and the acrylic plate got displaced and caused breakdown of total internal reflection. The scattered light rays from the white sheet were recorded by the webcam. Computer software on Java platform was developed to grab and analyse video data to display dynamic pressure distribution in artificial colour contours. Approximate pressure calibration was obtained using static and dynamic values obtained from subjects with normal and abnormal foot pressures. Time graphs of pressure at user chosen points were also provided. The developed system worked with satisfaction and is being used for clinical assessment regularly.
\end{abstract}

Keywords: Pedograph, Pedobarograph, Dynamic foot pressure, Total Internal Reflection

\section{INTRODUCTION}

Diabetic patients usually suffer from lack of nerve sensation, especially in the feet. Therefore, their gait on standing and during walking may deviate from that of a normal person and points of high pressure develop under the feet. However, due to neuropathy they do not feel any pain which would have been felt had the nerve functions were alright. Later, ulcers form at these high pressure points; gangrene and amputation of the leg follow suit. Therefore, a simple device to assess and measure the pressure distribution under the feet would be very helpful in early detection of high pressure points. Special insoles can then be prepared to spread the pressure away from the hot spots, thus saving the patient from eventually from the traumatic amputation crippling the person for the entire life. Again, pressure points and pattern may differ while standing and during walking, usually it is the latter which put the soles on greater burden, and a dynamic pressure measurement during walking has more importance from a clinical point of view.

Many attempts have been made over the last decades in measuring such pressure distributions under the feet. Simple ink impressions on a paper, permanent mechanical deformation created by a foot on specialized material, etc., have been used in the long past [1],[2]. However, they suffer from various disadvantages, the important one being the lack of a large dynamic range, lack of precision for distinguishing the pressures at different points, and requiring fresh materials for each test. In the later part of the last century several attempts have been made to make computerized systems using optical sensors for dynamic pressure measurement [3]. These typically used a clear glass slab placed horizontally with tube-light placed at an edge of the slab where light travels within the slab through total internal reflection. A still or a video camera is placed suitably below, mostly through a mirror placed at $45^{\circ}$, to photograph the underside of the feet pressing onto the glass plate. Some early models had a person standing on the glass plate and the camera taking a picture of the feet pressing onto the glass plate. Blood moved away from the high pressure points and this change the brightness and colour between pressurized and less 
pressurized areas. An improved version used a white paper or plastic sheet spread on the top and the person walks over this sheet. At points of pressure, air molecules between the sheet and the acrylic slab are removed which causes a breakdown of total internal reflection at those points. As a consequence light goes out of the slab at these points and are reflected and scattered by the white sheet. The video camera gets an image of these light patterns which essentially is a distribution of pressure. A further improvement was made by cutting off external light by placing a black sheet on top of the white sheet. However, none of these instruments are commercially available.

Recently commercial Pedograph, or Pedobarograph equipment have become available [4],[5] which use a matrix of small pressure transducers distributed over the whole sensitive area on which a person walks through. These provide a lower resolution compared to the above mentioned optical sensor, but may offer a higher accuracy in pressure transduction. However, these instruments, sold only as a complete package with computers and software are very expensive, and beyond affordability of most Third World clinics and hospitals. Reference [6] gives a review of different types of foot pressure measurement systems that have been made at different times.

Therefore an attempt was made to improvise and develop a low cost Pedograph, including sensor and computer software, suitable for the Third World. Giving due consideration of various factors, a Pedograph based on an optical sensor was the choice of our group. Previous Pedographs based on this concept used a high quality glass plate with high transparency of light. However, such glass plates are not available in most places of the Third World. Besides, the price of such glass materials is usually very high. Common glass plates that are available have a low transparency (typically a green tint) and are not suitable for this application, since light has to travel a length of the order of half a metre without much attenuation. The present work looked for an alternative to the transparent glass plate, and this alternative should be readily available in the Third World. Secondly early designs used very expensive video cameras and digitization hardware and software. This is because the camera has to be sensitive to very low light levels; the scattered light rays from the white paper sheet at points of pressure. However, it was felt that digital cameras - both still and video - have gone through a big change in the last decade or so, and a low priced video camera may suffice. However, as noted above, it should have a low light sensitivity. Besides, it also should give at least about 30 frames per second, to give a dynamic image necessary for the present application. The software should be able to grab individual frames from the chain of images created by the video camera, and convert these to a user-friendly artificial colour coded video. Besides, analyses of the variation of pressure at different points of the sole should be performed, results being presented in the form of user friendly time graphs.

\section{METHODS \& RESUlts}

Since there are several parts to this work, the methods and results are presented together for each part as follows.

\subsection{Sensor design, improvisation}

For improvisation at low cost, the first change affected was in the choice of the transparent plate. We went for a $12 \mathrm{~mm}$ thick transparent acrylic sheet which is very cheap compared to glass with similar transparency. The acrylic sheet used had a very good transparency, more than $90 \%$ as measured using a light meter. However, it is easily scratched which adds to the scattered light, so care needed to be exercised in cleaning, wiping it dry using rubber squeeze, and mounting it. Once correctly fixed and mounted, there is little or no chance of further scratching.

The main part of the sensor, as shown in Fig.1, is the thick transparent acrylic plate. All its edges are polished smooth. A sheet of white paper is placed on its top (glossy art paper, $120 \mathrm{gm} / \mathrm{m}^{2}$ ), with the backing of a black adhesive plastic sheet which remains on the top covering the whole sensor. The black plastic sheet provides both light shielding and physical strength to sustain the pressure and shearing forces 
from the feet of the patients, giving the white paper sheet a long usable life. Light from a tubular fluorescent lamp enters the plastic plate from a side and passes within the transparent plate through total

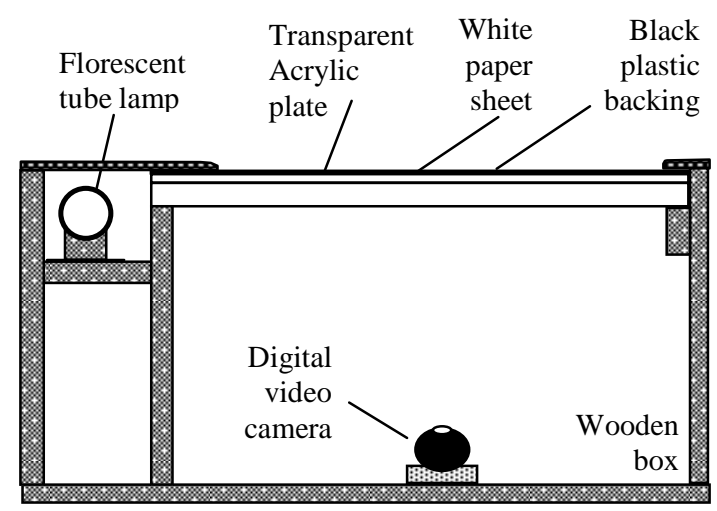

Fig.1: Schematic of the optical pressure sensor

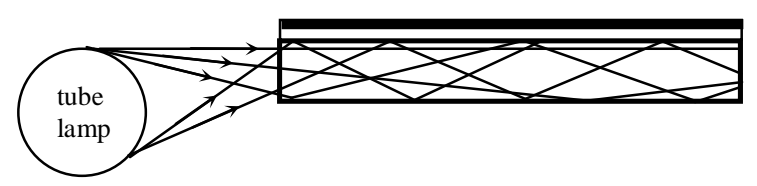

Fig.2: No pressure on sensor. Total internal reflection of all beams.

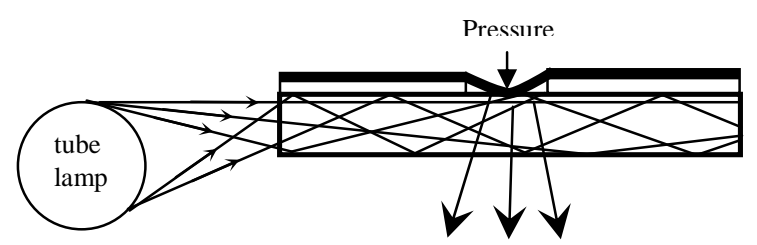

Fig.3. Pressure on sensor breaks down total internal reflection and scatters such beams downwards from the white paper sheet.

the edges and coming back at steeper angles (incident angle less than the critical angle) because of edge imperfections. At the edge where light enters, some beams emerging from the fluorescent lamp may have incident angles less than the critical angle, which will come out of the plate. To avoid these rays from going far in distance through multiple reflections, a matt black paper covers both the top and the bottom surfaces of the plate for a few inches near this edge.

The digital video camera takes successive photographs of the plastic plate on command from a Personal Computer (PC). The image-data is stored in internal reflection (Fig.2). Virtually no light comes out of the plastic sheet either upwards or downwards. A digital video camera is placed within the box underneath the plate facing upwards. The distance between the camera and the acrylic plate should be appropriate to focus the plate surface and cover the required field of view.

Normally without anything pressing onto the top of the sensing area, the camera essentially gives a dark background. At points where the foot presses onto the sensing area, the air in microscopic spots between the paper and the transparent plastic sheet are displaced. This causes breakdown of total internal reflection at these points and light rays go out of the acrylic plate upwards. There these rays are reflected and scattered by the white paper sheet From below, those points look as bright spots and the brightness increases with the amount of pressure as an increased pressure essentially increases the microscopic area of contact. The digital video camera placed below captures these patterns of light successively and stores the video data into computer memory. Later these data are processed to give an appropriate user friendly output described in the next subsection.

All the four edges of the acrylic plate are polished so that light is not scattered back from any of the edges. Three edges of the acrylic plate except the edge facing the fluorescent lamp are covered by matt black paper sheets to absorb light transmitted to the edges. This is to prevent light reflecting at

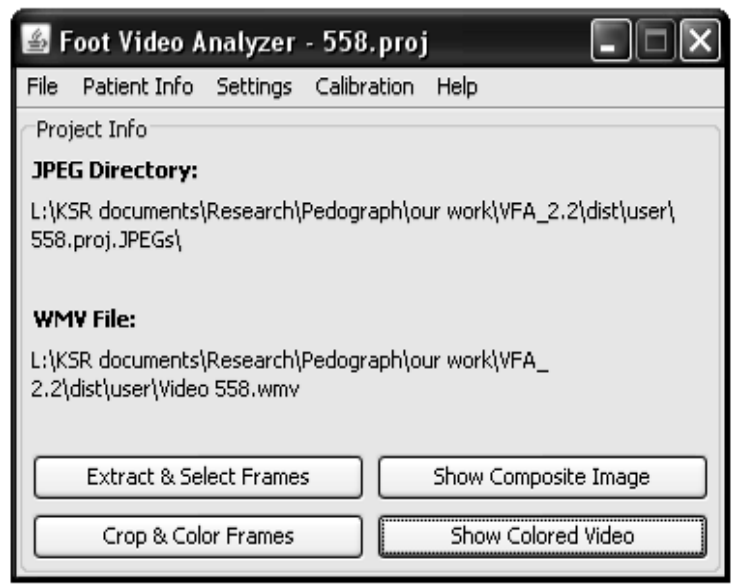

Fig.4. A view of the command window. The buttons appear in sequence to the command executed. 


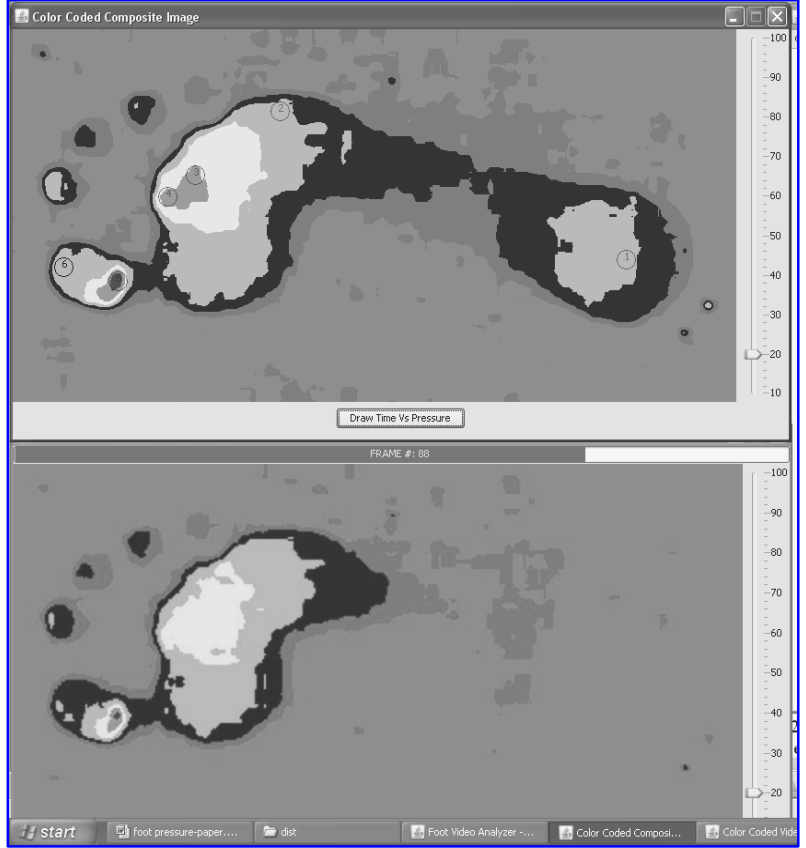

Fig. 5. Colour coded composite image (top) and a frame snap of the video (bottom) (reproduced here in monochrome)

the PC. To display the dynamic foot pressure while walking, a frame rate of more than 10 per second (fps) would be necessary, the greater the better. We targeted at $30 \mathrm{fps}$, and the camera should be sensitive enough to work at low light levels, since the scattered light due to pressure would ideally range from total darkness. Normal digital cameras have the required sensitivity and frame rates, but will require extra electro-mechanical gadgets to switch it on and off from outside. Extra steps would also be needed to acquire and store the video data in the PC. On the other hand Webcams can easily be controlled from a PC and the data is stored into the PC directly, and therefore have an advantage, but most of the available ones do not have the required frame rate at low light, as required by this application. Most of these webcams slow down frame rate at low light levels, giving more time for each exposure. After a thorough search only a few high end webcams were found that could satisfy the requirement, and one of these, a Logitech Pro 9000 was procured and used in the prototype. The video file is then read by the software developed in this project for analyses and display as described in the next section.

It needs to be mentioned that a flourescent tube light driven directly from the ac mains electricity is liable to give errors mainly due to two reasons: i) voltage fluctuations on the mains, and ii) regular sinusoidal variation at $50 / 60 \mathrm{~Hz}$. The latter will contribute to a change of light intensity at double the frequency. Since video data are recorded at $30 \mathrm{fps}$, the effect of the mains frequency variation may be significant. To eliminate this problem, a dc powered inverter operated at more than $20 \mathrm{kHz}$ was used. To keep the voltage reasonably constant a rechargeable battery on float charge was used for this power source.

\subsection{Computer display of results}

The software was developed in Java platform. On running the executable jar file a command window appears with a sequence of commands as shown in Fig. 4 which shows the final sequence. 'Extract \& Select Frames' is the first command to appear and clicking it leads to a browsing option to search for the video. On selecting the video file, the program creates frame by frame sequences and a composite image, all in gray scale. It then displays the image in frame sequences where the frame can be frozen, or run at slow or fast speeds in a movie mode, which continues repeating. The webcam chosen gives 30 frames per second images. Since the raw video can be for longer durations, unwanted images need to be discarded. Therefore, the programme prompts the user to select the first frame and to confirm. The programme automatically picks up the following 90 frames corresponding to 3 seconds of image data, assuming that the total time required by a foot step while walking would not be more than this, even for patients with foot problems. After confirmation, a 'Crop \& Color Frames' command appears. On selection, it displays a composite gray scale image and allows the user to crop it to the required size of interest, eliminating the background area. On confirmation the software prepares colour coded image data - frame by frame, and a composite one with the colours of the maximum value at all the pixels. 'Show Composite Image' and 'Show Coloured Video' are the two commands that appear next in sequence. Selecting each displays the respective images in two separate windows which can be moved around the screen for a desired 


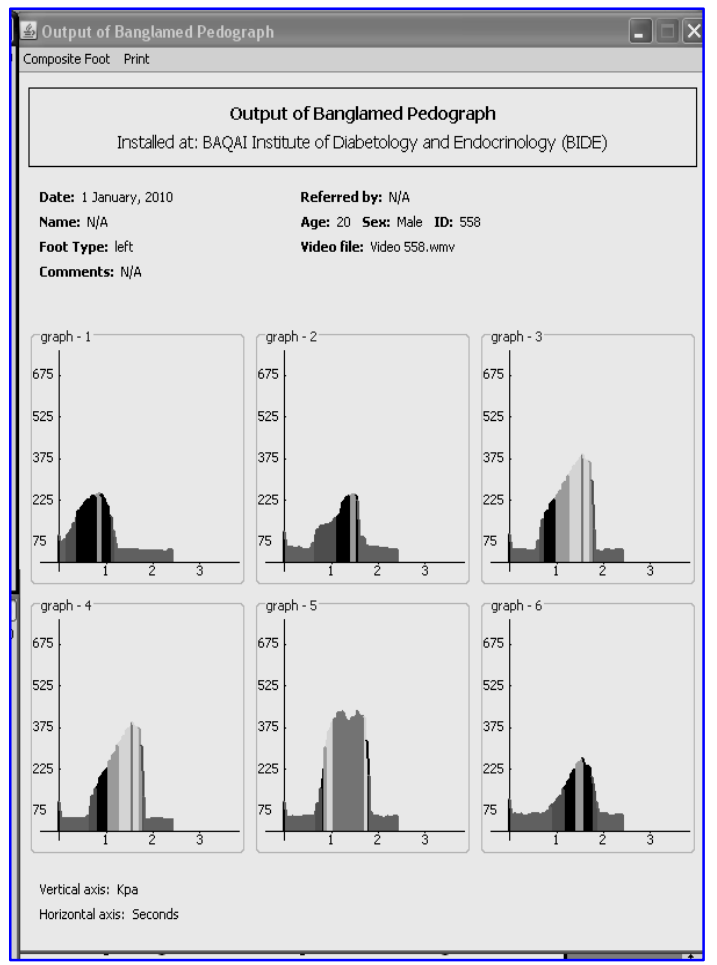

Fig. 6, Time graphs of pressure at 6 selected points as chosen on the composite image in Fig. 5 (indicated by placement. At this point the user can click up to 6 points on the composite image, or on the video, and click on another button to display time variation of pressure graph at the selected points. Each of these clicked points automatically creates a circular area around the point over which the average pressure is calculated. The size of the circle can be adjusted through a scale placed on the right hand side of the window, but a default value is given initially. On clicking the button to plot graph after the selection is made, a separate window appears with the time graphs as shown in Fig. 6. There is a choice between line graphs and solid filled graphs. The colour coding has been done to match a psychological viewpoint to represent the grading of pressure. The progression of colour chosen, in ascending order of pressure is, gray, light blue, deep blue, yellow, green, orange and red. On a tool bar in the graph window, clicking onto 'Composite Image' shows the composite image together with a colour coded calibration chart as shown in Fig. 7. Finally on selecting the 'Print' option in this window, this figure as well as the time graph window shown in Fig. 6, are printed out on paper. For the display, a measured background value is subtracted from all the data values before plotting the images and the time graphs. This non-zero background possibly occurs because of imperfections in the apparently transparent acrylic plate, which scatters some light.

\subsection{Calibration}

It has been reported that pressure sensitivity of optical sensors as used have different values for static and dynamic pressures, i.e., the sensitivity depends on the time of the pressure being applied [7]. This is an important issue which needs to be incorporated in the design of equipment. An attempt was made first to obtain a static calibration, and this was used as a guide to an approximate dynamic calibration attempted with actual dynamic foot pressure images from normal healthy persons, and from patients with severe diabetic neuropathy. For static calibration, two 3" x 6" wooden platforms having four legs each were made. The legs had circular cross sections with diameters of 1" and 0.7 " respectively for the two platforms. This makes the area of the former twice that of the latter. Thick rubber pads were attached to the legs to distribute the pressure to the whole contact area as much as possible. Such a platform placed on the sensitive area of the Pedograph sensor is shown in Fig. 8.

A person with a known weight stood on it with one foot only for a brief period. No other parts of the body including

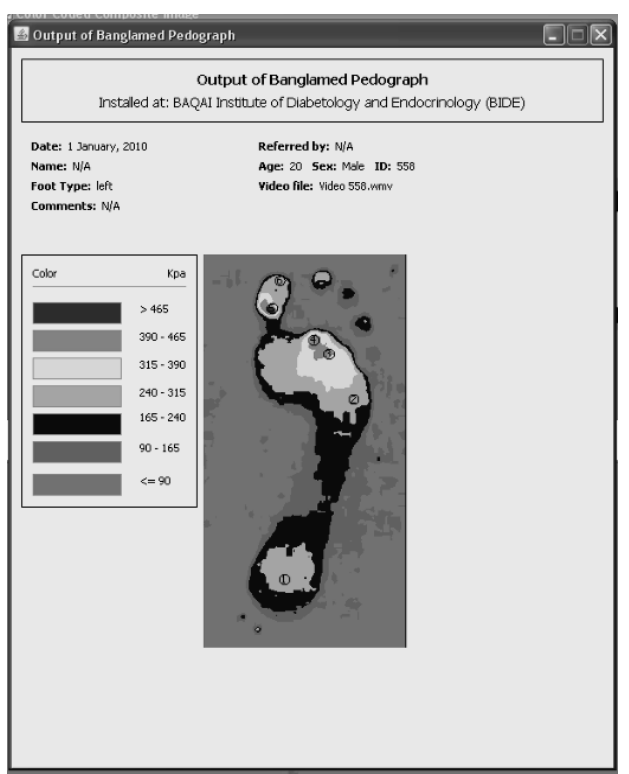

Fig. 7. Composite image with colour chart. This is also the printed version of the composite image (reproduced in monochrome). 
the other foot touched anything else. A video with the webcam was taken for a few seconds in this position. This was then analysed using the above mentioned foot pressure software, but with slight modifications. Instead of the pressure values, the raw pixel values that are directly proportional to brightness were plotted. In the processed image the raw pixel values varied between 0 and 255 . Four graphs corresponding to the four legs of the platform were obtained where the circular area of measurement were adjusted to match the leg contact area. Average pixel values obtained under the four legs and the corresponding pressures obtained from the weight and the total leg contact area were recorded. This scheme was repeated with the same person standing on the other platform. Similar measurements were obtained from persons with significantly different weights, which gave a range of pressure values. A linear plot was obtained with pixel values against pressure in $\mathrm{kPa}$ using the above data and a typical one is shown in Fig. 9.

It needs to be mentioned that the calibration depends on

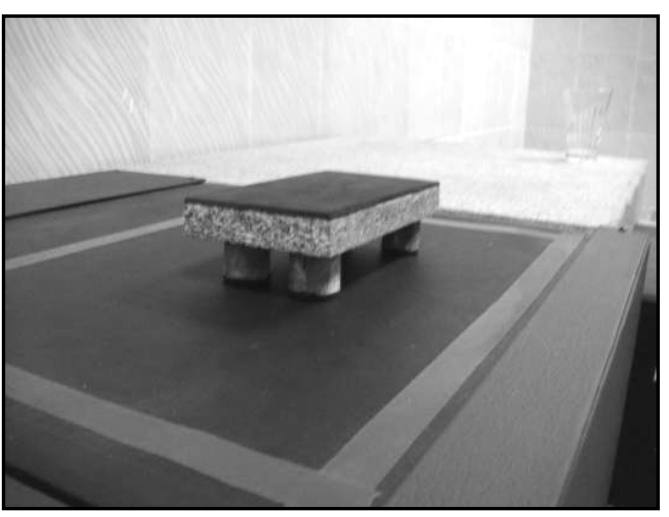

Fig. 8. Calibrating platform having four legs with circular cross sections and placed on the sensitive area of the Pedograph. the settings of the webcam. Any automatic setting will change the exposure depending on lighting conditions. Therefore, the webcam was set for all manuals. It can be seen that the measured data points may be fitted by a straight line, but the dynamic range is rather small. Values for brightness and contrast of the webcam allowed a good control over the pixel values and the dynamic range of the calibration curve. These were optimised after several trials, to get the highest dynamic range. These settings of the webcam were maintained for the dynamic foot pressure calibration described below.

For dynamic foot pressure calibration there are several difficulties. Since the brightness depends on the time that a pressure is applied for as mentioned above [7], it will change slightly with the speed of walking and will also be different for different points under the same foot. However, in the clinical setting, it is the 7 colour ranges that will guide the assessment; a precise pressure calibration may not be very important. Besides, a user would be more interested in the pressure over a small area rather than at individual pixels in the clinical setting which will again have an averaging effect. Therefore, an approximate calibration for dynamic foot pressure was attempted through several images obtained from normal persons, and from diabetic patients having high localised pressure areas under the feet. An approximate relationship with $\mathrm{kPa}$ values were obtained from reports that foot pressures above $500 \mathrm{kPa}$ are considered abnormal, although some reports quote widely different values [8]. The background where no pressure was applied showed a non-zero pixel value. This could be due to imperfections in the apparently transparent acrylic plate and was to be subtracted from all the pixel values in the image. However, to provide a background colour range of gray in the image, and to ensure that it was not over compensated, the

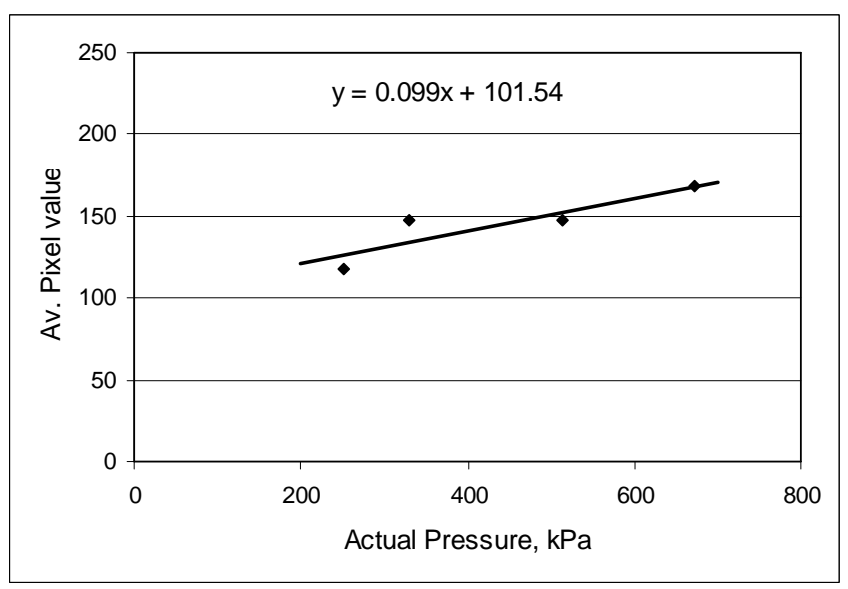

Fig. 9. A static calibration curve with data obtained from two persons. 
full amount was not subtracted. The images and graphs shown in Figs. 5 to 7 were obtained after such adjustments and calibration. This was later applied to study more subjects, both normal and abnormal, and the calibration relationships were adjusted accordingly. The results from subsequent clinical studies appeared to be satisfactory.

\subsection{Resolution}

The inherent resolution offered by this webcam is very high compared to the requirements. The camera is usually set to a resolution of $640 \times 480$ covering a field of view of $495 \mathrm{~mm} \times 370 \mathrm{~mm}$ at the top surface of the acrylic plate. This gives an area of about $0.6 \mathrm{sq}-\mathrm{mm}(0.78 \mathrm{~mm}$ square $)$ per pixel of image. The resolution will also depend on the thickness and elasticity of the white paper sheet and its adhesive plastic sheet backing. For the particular assembly used, it was found experimentally that a resolution better than 4 sq-mm ( $2 \mathrm{~mm}$ square), corresponding to 25 pixels per sq-cm, is obtained. A few commercial systems based on dot capacitors usually give a resolution of about 4 elements per sq-cm [4]. Therefore, this optical sensor has a much better resolution compared with such capacitor based systems.

\section{DISCUSSION}

This paper presents a practical approach to the development of a pedograph for a Third World setting, at a very low cost. The sensor is made mostly from items available in the Third World. Personal computers are widely available too. The only item that is not readily available is the high end webcam. However, the price, around $\$ 100$ per piece, is low enough to keep a stock of several units for fabrication.

The acrylic plate is easily scratched, and so needs careful handling and preparation. However, once it is installed further possibility of scratching is minimal. Acrylic plates obtained from different manufacturers were found to have different resistance to scratches, and their internal clearness was also slightly different. Therefore, one has to test the available ones to get the highest quality.

The quality of the white paper sheet is important. A matt sheet may scatter light too much and the image obtained in the camera would be dim. Therefore, a glossy sheet was used. Again the thickness should be optimal too. If it is too thin it may not spring back to its natural state quickly enough contributing to a loss of temporal information. The same applies to plastic sheets, most of which are sluggish in return. Again if the paper sheet is too thick it may spring back quickly after a pressure is removed but the resolution will be reduced as the pressure applied at any point will spread out a little. In the present work a $120 \mathrm{gm} / \mathrm{m}^{2}$ glossy art paper produced satisfactory results. The adhesive plastic sheet backing becomes integrated with the white paper sheet, so their combined elastic property comes into play. Therefore the plastic sheet should be as thin as possible. On the other hand it should be strong enough to bear the pressure and shearing forces due to patients walking, to last a reasonably long period. Addition of the plastic sheet is liable to reduce resolution, but that is not too much of concern, as reported earlier. The combined resolution was much better than that of expensive commercial systems based on dot capacitor sensors.

The intensity of light of the fluorescent tube lamp may gradually diminish with age. This may be compensated by adjusting the brightness of the webcam for a standard weight placed on the sensor area. The same is to be done when replacing a tube lamp.

The colour codes corresponding to different ranges of pressures were decided upon by the authors on discussion with the users, based on the local psychological reactions. It does not exactly follow codes used by similar commercial equipment.

The main drawback of this system is the lack of accurate pressure calibration, although for the clinical purpose targeted, this approximate calibration is reasonably satisfactory. As mentioned before, the static and dynamic pressure calibrations are different. This means that the light output will depend on the time 
of contact, which may differ from pixel to pixel, and for test to test, even for the same subject. Even existing commercial pedographs showed a large difference between absolute values of pressures measured [9]. Therefore, further work needs to be done to improve this aspect. Through carefully designed experiments the relation between pressure and the time of contact for each individual system has to be determined. This information may then be used to develop computer programmes to calibrate individual pixels based on its time of contact.

The prototype made has been used to obtain clinical evaluation of more than 150 patients to date and is still in a good condition. This shows the durability of the materials chosen in the design, and its practical use in the clinic.

\section{CONCLuSion}

This work shows that with newly available materials and devices, low cost improvisation of a Pedograph is possible that can help the delivery of modern healthcare to a wider population in the Third World.

\section{ACKNOWLEDGEMENT}

The work was carried out jointly by BIBEAT and the Department of Biomedical Physics \& Technology, University of Dhaka, Bangladesh. The authors would like to acknowledge Baqai Medical University in Karachi, Pakistan for funding this research project, and for using a prototype in their clinical test centre for gaining practical experience.

\section{REFERENCES}

1. WC Harris, T Beath. "Canadian Army Foot Survey," National Research Council, 1947

2. Ivo Waerlop, Shawn Allen, Robert Lardner. (2010) The Homunculus Group webpage on The pedograph as a window to the gait cycle. [Online]. Available: http://homunculusgroup.squarespace.com/pedo-article/

3. C. 1. Franks, R. P. Bens, and T. Duckworth, "Microprocessor based image processing system for dynamic foot pressure studies", Med. and Biol. Engg. And Comput. Vol. 21, pp. 566 - 572, 1983.

4. (2010) Novel gmbh website. [Online]. Available: http://novel.de/novelcontent/emed

5. (2010) Tekscan, Inc. website. [Online]. Available: http://www.tekscan.com/medical/system-matscan1.html

6. Stephen Urry, Plantar pressure-measurement sensors (Review article), Meas. Sci. Technol. Vol. 10, pp. R16R32, 1999.

7. C. I. Franks, "Calibration of optical foot pressure systems," Medical and Biological Engineering and Computing, vol. 35, pp. 69-72, Jan.1997, [Online]. DOI:10.1007/BF02510396

8. Marvin E. Levin, Lawrence W. O'Neal, John H. Bowker, Michael A. Pfeifer, Levin and O'Neal's the diabetic foot, Elsevier Health Sciences, 2008.

9. B Quaney, K Meyer, MW Cornwall, TGA McPoil, Comparison of the dynamic pedobarograph and EMED systems for measuring dynamic foot pressures, Foot Ankle Int. vol. 16, pp. 562-566, Sep.1995. 